\title{
Finding Useful Items and Links in Social and Agent Networks
}

\author{
Sandip Sen \\ University of Tulsa, USA \\ sandip-sen@utulsa.edu
}

\begin{abstract}
In this talk, I will overview our recent work addressing the problem of locating useful items and trading partners within large-scale social and agentorganized networks.

In agent-organized networks (AONs), agents have a limited number of collaboration partners at any time, represented by edges in a network of agent nodes. Agents have to learn to estimate the utility of current trading partners and rewire edges, i.e., change partners to improve profitability. We show that an exponentially decaying exploration scheme produces similar utilities with much less rewiring costs compared to a previously proposed random partner selection scheme within a production and exchange economy. We analyze the observed performance and demonstrate the robustness of our proposed rewiring scheme in a more realistic model of the economy that incorporate minimum trade volumes and storage capacities.

We have developed a Social Network-based Item Recommendation (SNIR) system consisting of agents that utilizes the connections of a user in the social network to search for items of interest posted by peers. Our approach enables a focused mining of the huge number of items available in the social network to generate targeted recommendations that can improve the precision of the result returned from a user's query. We have implemented the SNIR agent-based framework in Flickr, a photo-sharing social network, for searching and recommendation of photos by using tag lists as search queries. We discuss the architecture of our system, motivate the searching and referral schemes used, and demonstrate the effectiveness of the SNIR approach by presenting representative results.
\end{abstract}

